\title{
Strong Enhancement of Critical Current Density in MgB2 Bulk and Ni-Sheathed Tapes With Very Small Dy2O3 Additions
}

\begin{abstract}
A series of polycrystalline samples were prepared by in situ reaction of Dy2O3 (0.5-5.0 wt.\%) with $\mathrm{Mg}+\mathrm{B}$. While the superconducting transition temperature, Tc remained largely unchanged, the intragrain distribution of nano precipitates of $\mathrm{DyB} 4$ and $\mathrm{MgO}$ led to strong enhancement in critical current density, Jc at lower field below $4 \mathrm{~T}$. The best sample (with only 0.5 wt.\% Dy $2 \mathrm{O} 3$ additions) had a Jc (1 T) of around a factor of 4 higher compared to the pure sample at $6 \mathrm{~K}$ and $20 \mathrm{~K}$ indicating improved connectivity and pinning. On the other hand, ex situ Ni-sheathed tapes fabricated from the Dy2O3 added powders exhibited critical current, Ic $(4.2 \mathrm{~K})$ superior to those made from pure $\mathrm{MgB} 2$ powders at higher field above $3 \mathrm{~T}$. The former also showed lower anisotropy in Ic with respect to field direction.
\end{abstract}

Keyword: Critical current density, Dy2O3 additions, pinning. 